\title{
¿LA UNIVERSIDAD EN CHILE PROMUEVE LAS HABILIDADES DE ARGUMENTACIÓN ESCRITA? UN ESTUDIO EXPLORATORIO COMPARATIVO DE ESTUDIANTES DE EDUCACIÓN UNIVERSITARIA Y EDUCACIÓN TÉCNICA
}

\author{
Antonia Larraín, Paulina Freire, Renato Moretti, \\ Magdalena Requena y Belén Sabat ${ }^{1}$
}

RESUMEN

Las habilidades de argumentación juegan un rol central en la formación universitaria. Evidencia disponible muestra que los estudiantes universitarios cuentan con mejores habilidades argumentativas que sus pares no universitarios. Sin embargo, no se sabe si esto se debe a su formación o si es un factor de autoselección. El objetivo de este estudio fue explorar el desarrollo de habilidades de argumentación en estudiantes de educación superior, de manera de contribuir al conocimiento acerca de la relación entre argumentación y educación superior (universitaria y técnico profesional) en Chile. Se evaluaron las habilidades de argumentación escrita de 336 estudiantes de educación universitaria y educación superior técnica, provenientes de cinco carreras diferentes de dos instituciones de educación superior de Santiago Chile, y dos cohortes distintas, al inicio y al final del año académico. Se realizaron análisis descriptivos, correlacionales y análisis de covarianza. Los resultados muestran que los participantes presentan debilidades relativas en la capacidad de formular contra-argumentos; que en general avanzan significativamente en los sub-aspectos de aceptabilidad y suficiencia argumental; y que si se los controla por edad y medidas de rendimiento, los estudiantes universitarios avanzan en un año significativamente más que los de educación técnica. Por último, los resultados muestran que los alumnos universitarios que más avanzan son los que están bajo el promedio en la Prueba de Selección Universitaria (PSU). Se discuten las implicancias de estos resultados para la educación superior en Chile.

Palabras clave: educación superior, argumentación, desarrollo cognitivo.

\section{UNIVERSITY AND ARGUMENTATION SKILLS' DEVELOPMENT IN CHILE: AN EXPLORATORY AND COMPARATIVE STUDY}

ABSTRACT

Argumentation skills play an important role in higher education. Empirical evidence shows that higher education students have better argumentation skills than their peers without university studies. However, it is still unknown whether this is a product of higher education or self-selection. The goal of this study was to explore written argumentation skills development in Chilean tertiary education, in order to contribute to the body of knowledge on the relationship between argumentation and higher education (university and vocational training). The written argumentation skills of 336 tertiary education students, from university and vocational training programs, were evaluated. The sample was comprised of five different programs from two Chilean institutions, and of two cohorts, evaluated at the beginning and end of the school year. Descriptive, correlational and covariance analyses were run. Results show that the participants are less skilled at formulating counter-arguments that in general, they significantly improve in the sub-aspects of acceptability and soundness; and when controlled for age and academic performance, university students improve significantly more than technical students, over the course of one year. Finally, results show that university students' improvement is greater for those that have below average performance on the standardized test required for university admission in Chile (PSU). The implications for Chilean higher education is discussed.

Keywords: higher education, argumentation, cognitive development

1 Todos los autores pertenecen a la Facultad de Psicología de la Universidad Alberto Hurtado, Santiago, Chile. Contacto: alarrain@uahurtado.cl 


\section{Argumentación y universidad}

Si pensamos que la universidad tiene como misión central la producción y reproducción de conocimiento especializado, podemos pensar que las habilidades de producción de este conocimiento, entre las que se encuentran las así llamadas habilidades superiores de pensamiento, son habilidades transversales que la universidad debe desarrollar, más allá de las condiciones de ingreso de los estudiantes y de las especificidades disciplinares. De hecho, aunque las instituciones universitarias entregan formación profesional, la dimensión reflexiva del trabajo universitario sigue siendo lo más peculiar de la universidad (Peña, 2008), caracterizando con ello la calidad de un proceso de educación universitaria (Terenzini, 2010; Pascarella y Terenzini, 1991). Según Carlino (2005) el estudiante universitario llega a pertenecer a una comunidad científica y/o profesional, en virtud de haberse apropiado de las formas de razonamiento propias de esa comunidad instituidas en prácticas discursivas.

Un considerable número de investigaciones ha tenido como propósito evaluar el grado en que los estudiantes que asisten a la universidad se desarrollan cognitivamente más que las personas que no asisten a ella. En su gran mayoría, la evidencia sugiere que la asistencia a la universidad contribuye de manera significativa al aprendizaje y desarrollo cognitivo, más allá de lo esperado por la maduración y experiencias de vida (Pascarella y Terenzini, 1991, 2005). La evidencia sugiere además que los estudiantes que corresponden a la primera generación que accede a la universidad, se benefician de la experiencia universitaria a pesar de tener condiciones de entrada desventajosas; que esta experiencia es diferente a la de los estudiantes tradicionales (Terenzini, Springer, Yaeger, Pascarella y Nora, 1996); y que los primeros años de educación universitaria corresponden al período en el que se produce el mayor nivel de ganancia cognitiva en los estudiantes universitarios (Pascarella y Terenzini, 2005; Reason, Terenzini y Domingo, 2006).

Dentro de las habilidades cognitivas, las de razonamiento y, con ellas, las de argumentación, juegan un rol central (Mercier y Sperber, 2011). De ahí, entonces, que la enseñanza universitaria tenga 
una responsabilidad ineludible en la promoción de habilidades de razonamiento y argumentación. Así lo reconoce el proceso de Boloña (Mouraz, Leite, Trindade, Martins, Faustino y Villate, 2014). De hecho, las habilidades de argumentación tienen un peso central en las evaluaciones universitarias, sobre todo en los primeros años (Andrews, Bilbro, Mitchell, Peake, Prior, Robinson y Togerson, 2006). Sin embargo, este peso parece ser fruto de prácticas pedagógicas que dan por supuesto las habilidades argumentativas, pero que escasamente la enseñan (Andrews et al., 2006). La universidad que enseña a estudiantes con altos niveles de argumentación y razonamiento -niveles que desarrollan previa o paralelamente en su educación escolar o experiencias familiares-, no corresponde a la universidad masificada contemporánea. Si el razonamiento es un requisito central de un profesional o licenciado universitario, este debe ser enseñado.

Por otra parte, existen múltiples maneras de concebir la argumentación (Leitão, 2009). Aquí la entenderemos como la actividad social y discursiva de lidiar con opiniones controversiales, con el objeto de alcanzar un mayor entendimiento, ya sea entre dos personas que sostienen distintos puntos de vista, o bien, entre dos puntos de vista sostenidos por un mismo hablante. En este sentido, la argumentación involucraría la justificación de los puntos de vista y/o su evaluación crítica, coordinando evidencia teórica y/o empírica.

Ahora bien, la argumentación no se da en un vacío. Por una parte, para que esta emerja se requiere la presencia de mecanismos semióticos específicos que convierten una pieza discursiva en argumentativa (Leitão, 2000a). A su vez, para que se usen mecanismos semióticos propios de la argumentación se requieren ciertas características particulares del contexto: la argumentación es muy sensible al carácter polémico del tema, al tipo de instrucciones, a la distribución de poder entre los participantes y al conocimiento o familiaridad con el tema, entre otros aspectos (Coirier, Andriessen y Chanquoy, 1999; Glassner y Schwarz, 2005; Golder y Pouit, 1999; Leitão, 2000a, 2000b; Santos y Santos, 1999). Por otra parte, toda interacción argumentativa o proceso de argumentación, a la vez que promueve habilidades argumentativas en los participantes (Kuhn y Crowell, 2011), depende de ellas. 
Las habilidades argumentativas pueden entenderse en términos de la calidad del desempeño que muestran los hablantes en la construcción y evaluación de argumentos en situaciones argumentativas. Aunque la discusión acerca de la calidad de la argumentación es difícil e involucra múltiples aristas (O’Keefe, 1995), Govier (2010) ofrece criterios simples y útiles para evaluar la calidad de una construcción argumentativa. Ella plantea que un argumento de calidad es un argumento cogente, esto es, que cumple con tres condiciones: sus premisas son aceptables, relevantes y suficientes para sostener la conclusión. En términos de su capacidad persuasiva, es importante considerar que un buen argumento es, además, uno que anticipa y responde potenciales contraargumentos (Golder 1992, citado en Golder y Pouit, 1999). Esta es justamente la debilidad más documentada: los hablantes tienden a mostrar lo que se ha llamado el sesgo de justificación, es decir, tienden a formular apoyos y justificaciones para la propia postura en mayor medida que anticipar y responder abiertamente a las debilidades.

Las investigaciones respecto del desarrollo de la argumentación sugieren que si bien los hablantes argumentan desde muy pequeños (Stein y Albro, 2001; Stein y Miller, 1993), el desarrollo de estas habilidades no está garantizado ni obedece a una programación universal: la medida en que los niños desarrollan habilidades de argumentación dependerá de la cantidad y calidad de las experiencias en las que estos tengan que argumentar. En otras palabras, las habilidades individuales de argumentación se desarrollan argumentando con otros. Esto ha sido ampliamente estudiado y la evidencia empírica muestra clara y consistentemente que los estudiantes mejoran sus habilidades de argumentación individual (la mayoría a través de mediciones escritas), luego de la participación sistemática en contextos en los que se argumenta y se fomenta la argumentación, independientemente de si se enseña de forma explícita a hacerlo o no, o de la edad de los participantes (Belland, 2010; Chen y She, 2012; De Chiaro y Leitão, 2005; Garcia Mila y Andersen, 2008; Kuhn y Crowell, 2011; Kuhn y Udell, 2003; Pirchio y Pontecorvo, 1997; Pontecorvo y Pirchio, 2000; Rojas-Dummond y Peon, 2004; Song y Ferreti, 2013; Sunal, Sunal y Tirri, 2001; Walker, Wartenberg y Winner, 2013; Wilson, Taylor, Kowalski y Carlson, 2010; Zohar y Nemet, 2002). 
Por su parte, si bien las habilidades argumentativas aparecen tempranamente, los niños van en forma progresiva siendo más capaces de elaborar argumentaciones más complejas (Stein y Bernas, 1999). Primero, solo elaboran puntos de vista, luego son capaces de apoyarlos con simples "sî" o "no" y, posteriormente, son capaces de desarrollar apoyos más complejos. La elaboración de contraargumentos, relacionada con la posibilidad de percibir tensiones, aparece como un logro posterior del desarrollo, sobre todo si los contra-argumentos son dirigidos al propio punto de vista.

Respecto de la argumentación escrita, se puede afirmar que su desarrollo, si bien está íntimamente relacionado con la argumentación oral, depende también del manejo de lenguaje escrito general que tenga un hablante, así como de las oportunidades que tenga de argumentar de manera escrita (Piolat, Rousseay y Gombert, 1999). Para argumentar de manera escrita saber escribir es necesario, pero no suficiente (al igual que para argumentar oralmente es necesario saber hablar, pero no suficiente); se requiere aprender a escribir argumentativamente. De hecho, la investigación sugiere que un niño de ocho años con entrenamiento en esquemas argumentativos argumentará igual que un niño de doce sin ese entrenamiento. Del mismo modo, si bien el inicio del desarrollo de la argumentación escrita es más tardío que el de la argumentación oral (pues depende de la capacidad de escribir relatos coherentes), el desarrollo de ambas tiene efectos potenciadores (Stein y Bernas, 1999). En otras palabras, el desarrollo de la argumentación oral se verá favorecido por el entrenamiento en argumentación escrita y viceversa.

Un clásico estudio de Kuhn (1996) en el que participaron 160 personas agrupadas en cuatro cohortes de edad (escolares adolescentes, adultos jóvenes y adultos de 40 y 60 años), y en el que se midieron las habilidades de argumentación oral a través de entrevistas, sugiere que estas se desarrollan de acuerdo con la edad hasta la adolescencia. Después de este momento las diferencias que muestran los jóvenes son producto de las experiencias de participación en actividades asociadas con la escolaridad. Los jóvenes universitarios muestran mejores habilidades argumentativas que los jóvenes adolescentes, pero un joven que no va a la universidad puede mostrar habilidades 
similares a estudiantes secundarios. Incluso más, la autora muestra cómo los estudiantes difieren en su habilidad argumentativa según provengan de establecimientos educacionales con ambientes sociales y académicos enriquecidos.

La evidencia disponible muestra que las habilidades argumentativas de los estudiantes secundarios son más bien bajas. Un estudio de Scholts, Sadek, Hodges, Lubben y Braund (2006), donde se midió la habilidad argumentativa espontánea de 266 estudiantes de educación media de colegios sudafricanos, obtuvo que un $85 \%$ de ellos presentaba niveles básicos de argumentación (de cinco niveles, donde el 1 era el más precario y el 5 el más avanzado; estos estudiantes se ubicaron entre los niveles 1 y 2$)^{2}$. En el caso de los estudiantes universitarios chilenos, los resultados del ensayo argumentativo que rinden los alumnos de la Pontificia Universidad Católica desde el año 2003 muestran que si bien estos obtienen en promedio un desempeño bueno en la calidad argumentativa del texto, la elaboración de contraargumentos solo alcanza en promedio un desempeño aceptable (Manzi y Flotts, 2012). La evidencia sugiere que este ensayo tiene una correlación estadísticamente significativa con el puntaje PSU promedio y el de la prueba PSU de Lenguaje, y que predice significativamente el desempeño académico de los estudiantes, incluso después de primer año, luego de controlar variables socioeconómicas y puntajes PSU, especialmente en Matemática (Preiss, Castillo, Grigorenko y Manzi, 2013). Por último, luego de controlar por variables de entrada, el ensayo tiene una relación sistemática con otras medidas de argumentación (Preiss, Castillo, Flotts y San Martín, 2013).

Si es que no hay evidencia de que la universidad enseñe a argumentar, ¿por qué los estudiantes universitarios tendrían más y mejores habilidades de argumentación que los estudiantes secundarios? Dada la relación mencionada entre PSU y puntajes en ensayos argumentativos escritos, una alternativa sería pensar que estas habilidades no se desarrollan durante la educación universitaria, sino que actúan como filtro para su acceso. Sin embargo, llama la atención

2 Evidencia chilena de estudiantes secundarios que apoya esta visión puede encontrarse en Marinkovic, 2007. 
que este ensayo tenga poder predictivo del rendimiento académico aún después de controlar por variables de entrada. Es posible que esto se deba al mencionado rol que tienen las habilidades argumentativas en las evaluaciones universitarias. Consecuentemente, otra alternativa es que aunque en la universidad no se enseñe de manera explícita a argumentar, la experiencia universitaria, particularmente aquella adquirida en evaluación de aprendizajes, no solo se vea facilitada por habilidades iniciales argumentativas altas, sino que promueva su desarrollo manteniendo una brecha entre quienes inicialmente muestran un mejor desempeño y quienes muestran un peor ejercicio en argumentación escrita.

El presente estudio se propuso explorar la pregunta por el desarrollo de la argumentación en la formación universitaria, de manera de contribuir al conocimiento acerca de la poco estudiada relación entre argumentación y educación universitaria en Chile. Particularmente, la pregunta que orientó el estudio fue si la formación universitaria promueve el desarrollo de la argumentación más allá de las condiciones de entrada de los estudiantes y en mayor medida que otras actividades educacionales superiores no universitarias. El objetivo general del estudio fue evaluar la relación entre la formación universitaria y el desarrollo de habilidades de argumentación escrita.

\section{Método}

Se realizó un estudio exploratorio con enfoque cuantitativo y diseño secuencial, en el que se evaluó la argumentación escrita de jóvenes participantes de formación universitaria (universidad) y de formación técnica (instituto), provenientes de diferentes carreras. Se compararon estudiantes universitarios y estudiantes de institutos de formación técnica para explorar si es que la universidad tenía un efecto específico en el desarrollo de las habilidades de argumentación de los estudiantes, es decir, si tenía un efecto distinto del que tendría otra experiencia de educación superior no universitaria (en este caso técnica). Se evaluaron dos cohortes de formación (primer y segundo año) al comenzar y al finalizar un año académico. 


\subsection{Participantes}

Los participantes fueron estudiantes de primer y segundo año de tres carreras universitarias (Psicología, Derecho e Ingeniería Comercial) y dos carreras técnicas (Técnico Jurídico y Asistente de Párvulos). La universidad es una institución privada no perteneciente al CRUCH, ubicada en Santiago centro, al igual que el instituto profesional. De un universo inicial de 584 estudiantes de estos años y carreras que estuvieron presentes en las salas al momento de realizar la recolección de datos, 469 (82,5\%) finalmente aceptaron participar entregando su consentimiento por escrito. De este grupo 143 (23 hombres) eran de la educación técnica y 326 (156 hombres) de educación universitaria. De este universo total 456 estudiantes participaron, o bien respondiendo solo el cuestionario (120), o el cuestionario y al menos un ensayo argumentativo (336). En la Tabla 1 se observa la composición de la muestra considerando la distribución de carreras y sexo, mientras que en la Tabla 2 se observa la distribución por sexo según el año de estudio de los estudiantes que respondieron el cuestionario.

Tabla 1: Distribución sexo según carrera participantes con consentimiento

\begin{tabular}{|l|c|c|c}
\hline \multirow{2}{*}{} & \multicolumn{2}{|c|}{ Sexo } & \multirow{2}{*}{ Total } \\
\cline { 2 - 3 } & Hombre & Mujer & 117 \\
\hline Derecho & 67 & 50 & 98 \\
\hline Ingeniería Comercial & 52 & 46 & 111 \\
\hline Psicología & 37 & 74 & 75 \\
\hline Asistente de Párvulos & 0 & 75 & 68 \\
\hline Asistente Jurídico & 23 & 45 & 469 \\
\hline Total & 179 & 290 & \\
\hline
\end{tabular}

Tabla 2: Distribución sexo según año de estudios, participantes que respondieron cuestionario

\begin{tabular}{l|l|c|c|c}
\hline \multicolumn{2}{c|}{} & \multicolumn{2}{|c|}{ Sexo } & \multirow{2}{*}{ Total } \\
\cline { 3 - 4 } \multicolumn{2}{c|}{} & Hombre & Mujer & 270 \\
\hline \multirow{2}{*}{ Año } & Primero & 101 & 169 & 186 \\
\cline { 2 - 5 } & Segundo & 69 & 117 & 456 \\
\hline Total & 170 & 286 & \\
\hline
\end{tabular}


Respecto de la composición por sexo de la muestra, hay diferencias significativas, donde destacan las carreras de Psicología y Asistente de Párvulos, esta última compuesta netamente por mujeres. El grupo instituto presenta un $82,4 \%$ de mujeres contra un $54,2 \%$ del grupo universidad. Por otra parte, las carreras técnicas tienen una media de edad significativamente mayor que las carreras universitarias. De manera coherente, los participantes de la universidad egresaron en torno al año 2009 ( $X=2009,32 ; D E=2,56)$, mientras que los participantes del instituto lo hicieron alrededor de 2007, con una desviación estándar mayor $(X=2007,05 ; D E=3,11)$.

\subsection{Procedimiento}

En primer lugar se contactó a los directivos de un conjunto de carreras de una universidad y de un instituto profesional que ofrece carreras técnicas, para invitarles a participar en el estudio. La muestra se configuró entre las carreras que confirmaron su interés. Los directivos de carreras señalaron en qué horarios se podía realizar la medición. Se contactó a los docentes de las asignaturas y se acordó asistir a los últimos 10 minutos de clases para invitar al estudio y realizar la aplicación. Dos integrantes del equipo de investigación asistieron en el horario acordado a los cursos, informaron de los objetivos del estudio, en qué consistía su colaboración e invitaron a participar a los estudiantes. Aquellos que decidieron hacerlo firmaron una carta de consentimiento informado y respondieron las pruebas. Las primeras aplicaciones ocurrieron a comienzo del año académico 2012 (marzomayo), en tanto que las segundas sucedieron durante los meses finales (octubre-noviembre) del mismo año. Todos los estudiantes que participaron en el estudio rindieron un ensayo argumentativo. Se esperaba que rindieran el ensayo al inicio y al término del año, pero solo 102 participantes lo hicieron en ambas oportunidades, habiendo algunos que lo rindieron solo al inicio (226) y otros que solo lo dieron al final de año (108). En la Tabla 3 se resume la cantidad de estudiantes que rindió cada ensayo por carrera y en la Tabla 4 por año. 
210 ¿LA UNIVERSIDAD EN CHILE PROMUEVE LAS HABILIDADES DE ARGUMENTACIÓN ESCRITA? UN ESTUDIO EXPLORATORIO COMPARATIVO DE ESTUDIANTES DE EDUCACIÓN UNIVERSITARIA Y EDUCACIÓN TÉCNICA - A. Larraín, P. Freire, R. Moretti, M.Requena y B. Sabat

Tabla 3: Cantidad de estudiantes que responden cada ensayo por carrera

\begin{tabular}{l|c|c|c}
\hline Carrera & Ensayo 1 & Ensayo 2 & Ensayo 1 y 2 \\
\hline Derecho & 47 & 27 & 24 \\
\hline Ingeniería Comercial & 41 & 30 & 26 \\
\hline Psicología & 64 & 26 & 19 \\
\hline Asistente de Párvulos & 35 & 12 & 22 \\
\hline Asistente Jurídico & 39 & 13 & 11 \\
\hline Total & 226 & 108 & 102 \\
\hline
\end{tabular}

Tabla 4: Cantidad de estudiantes que responden cada ensayo por año

\begin{tabular}{l|c|c|c}
\hline Año & Ensayo 1 & Ensayo 2 & Ensayo 1 y 2 \\
\hline Primero & 129 & 54 & 58 \\
\hline Segundo & 97 & 54 & 44 \\
\hline Total & 226 & 108 & 102 \\
\hline
\end{tabular}

Al finalizar el proceso de investigación, se realizó una devolución de resultados grupales y confidenciales a cada carrera, con la finalidad de que estas contaran con conocimiento acerca del nivel de desempeño y desarrollo en argumentación durante los primeros años de formación de sus estudiantes.

\subsection{Instrumentos}

Se recurrió a los siguientes instrumentos.

\subsubsection{Cuestionario de caracterización}

Este instrumento consistió en un cuestionario de autorreporte de caracterización sociodemográfica desarrollado por el equipo de investigación, de manera de conocer datos clave de los participantes como puntajes PSU, dependencia educativa escolar, actividad de los padres y comunas de procedencia, entre otros.

\subsubsection{Ensayo de argumentación escrita}

Consistió en un ensayo de opinión cuyo objetivo fue evaluar la habilidad de argumentación escrita en jóvenes estudiantes de educación superior. Informados en la literatura de desarrollo de 
la argumentación y evaluación de argumentación escrita, y en experiencias nacionales en medición de esta habilidad, se decidió evaluar dicha habilidad a través de la escritura de un ensayo de opinión breve, que reuniera las siguientes condiciones:

- Instrucciones explícitas que demandaran desarrollar una opinión y dar razones tanto en pro como en contra.

- Tema polémico en el que se pueden sostener opiniones diversas sin sesgo.

- Tema que motivara a los participantes a dedicar tiempo y esfuerzo en mostrar sus puntos de vista.

- Tema que no involucre conocimiento especializado.

- Objetivo orientado a desarrollar una idea y no a convencer, para disminuir sesgo de justificación.

- Formulación explícita de opiniones contrarias en estímulo, para disminuir sesgo de justificación.

De esta manera, y siguiendo la experiencia en evaluación de producción escrita del Centro de Medición MIDE UC, se diseñó un instrumento que respondió y cuidó los aspectos antes mencionados. Un ejemplo de temas ofrecidos es el siguiente:

Algunas personas piensan que para tener éxito en la vida es fundamental esforzarse al máximo y aprovechar las oportunidades que se presenten. Por algo existe el dicho popular de "A quien madruga, Dios lo ayuda". De hecho, datos de la encuesta Casen apoyan la idea de que a más años de escolaridad, mayor ingreso, sobre todo si se tiene estudios superiores. Pero otros piensan que en un país como Chile más valen los contactos y pitutos que el mérito personal. Se cree que alguien que nació en una familia pobre, por mucho que se esfuerce, no logrará salir adelante. De hecho, datos de un estudio de la Universidad de Chile muestran que las personas que tienen "buenos" apellidos ganan más dinero por el mismo trabajo.

Las instrucciones planteaban que se estaba trabajando en una política de desarrollo de igualdad de oportunidades, ante lo que se animaba a los estudiantes a desarrollar su opinión, fundamentando y considerando tanto aspectos que apoyaran como aspectos que contradijeran su postura. Se realizó una aplicación piloto a 26 
estudiantes tanto de educación universitaria como educación técnica superior. La consistencia interna del instrumento final fue buena $(\mathbb{\Xi}$ $=0,743)$.

Se desarrolló una rúbrica de evaluación de cuatro niveles de desempeño (0 a 3 puntos). A diferencia de la prueba de MIDE UC, el interés fue minimizar la evaluación del componente de producción escrita, por lo que no se consideraron elementos de este tipo (ortografía, redacción, coherencia, caligrafía, etc.), más allá de la coherencia mínima para entender los planteamientos. El interés era obtener una medición de habilidades argumentativas lo más depurada posible de elementos propios de la escritura. Siguiendo los trabajos de Leitão (2000a) y Govier (1999), se consideraron dos aspectos de la producción argumentativa: estructura (sub-aspectos: planteamiento de posición, fundamentación y contra-argumentación) y calidad (aceptabilidad, relevancia y suficiencia):

- Planteamiento o formulación de una posición: se plantea claramente una posición o conclusión.

- Fundamentación: cantidad y articulación de premisas y subpremisas formuladas en apoyo a la conclusión.

- Contra-argumentación: formulación de argumento que debilita la relación entre premisas y conclusión, y se responde a este.

- Aceptabilidad: grado en el que las premisas esgrimidas tanto a favor como en contra de una conclusión son aceptables según el conocimiento disponible del evaluador.

- Relevancia: grado en el que las premisas aumentan la posibilidad de que la conclusión sea aceptada.

- Suficiencia: grado en el que el conjunto de premisas son suficientes para afirmar una conclusión.

Además, la rúbrica considera un ítem de articulación y cohesión de ideas.

Dos codificadoras especialmente entrenadas corrigieron los ensayos. En un primer momento se realizaron seis rondas de doble corrección con supervisión, discutiendo los desacuerdos y analizando ejemplos de cada uno de los criterios, de manera de enriquecer 
la rúbrica de corrección. En la última ronda de doble corrección supervisada (18,3\% del total), todos los ítems presentaban una confiabilidad interjueces muy buena (dos subaspectos $K>1$; dos $K>$ 0,8 ; y tres $K>0,71$ ). Luego, las dos codificadoras dobles corrigieron independientemente la totalidad de los ensayos. En un 89,9\% de los casos los desacuerdos fueron menores a un punto en el puntaje total de los ensayos. En los casos en los que los desacuerdos fueron mayores a dicha cifra, los ensayos fueron corregidos por la supervisora. El puntaje total se calculó promediando los puntajes de los dos evaluadores en cada subaspecto (en el caso de una tercera corrección por parte de los evaluadores más cercanos) y luego sumando la totalidad de subaspectos.

\subsection{Plan de análisis}

Con el fin de observar el desempeño de los estudiantes se realizaron análisis descriptivos. Para identificar las diferencias significativas entre los grupos se realizaron análisis de varianza y covarianza. Del mismo modo, se verificaron análisis correlacionales para observar el grado de relación entre las variables de interés.

\section{Resultados}

A continuación se presentan los principales resultados del presente estudio.

\subsection{Niveles iniciales de logro en argumentación}

Respecto del nivel inicial de habilidades de argumentación escrita que presentaron los estudiantes al ingresar a la educación superior, los resultados del análisis de frecuencia mostraron que una amplia mayoría en ambas instituciones es capaz de formular con claridad una posición relevante al debate (78\%), siendo mínima la diferencia entre los establecimientos. En relación con la fundamentación, aunque la mayoría de los participantes de ambas instituciones menciona más de una razón a favor de una posición y las articula, un porcentaje no despreciable no plantea razones a favor de su posición (6,7\%). El porcentaje de participantes con el mayor nivel de logro corresponde a universitarios (57,9\% versus $29,9 \%$ ). Coherentemente con lo que 
sugiere la literatura en argumentación, los participantes muestran deficiencias a nivel de contra-argumentación. Más de la mitad de los participantes no incorpora ningún tipo de objeción. Aunque en el instituto el porcentaje es mayor, se podría afirmar que la diferencia es muy leve respecto de la universidad. Por otro lado, solo un poco más de un $11 \%$ en ambas instituciones formulan contra-argumentos y los responden.

La mayoría de los participantes es capaz de formular premisas aceptables, pero no en su totalidad (54,8\%). Aunque el porcentaje de estudiantes que alcanza el nivel de logro intermedio es similar en la universidad y en el instituto, aquellos que alcanzan un mayor nivel de logro son porcentualmente más universitarios (28,9\%) en relación con los del instituto (10,2\%). La mayoría de los participantes es capaz de formular premisas relevantes $(42,6 \%)$. Sin embargo, los estudiantes universitarios, en un porcentaje considerablemente mayor, elaboran argumentos que contienen solo premisas relevantes (47,5\% versus $10 \%$ estudiantes de instituto). En el caso de la suficiencia de premisas llama la atención que solo un 2,74\% de los participantes formula premisas que son suficientes para aceptar la conclusión o punto de vista. La mayoría de los participantes muestra problemas de suficiencia tanto a nivel de premisas como de subpremisas $(47,8 \%)$. Nuevamente, un alto porcentaje de participantes de instituto presenta serias deficiencias en este ámbito, aunque ambas instituciones aparecen relativamente parejas.

\subsubsection{Ganancias en argumentación}

Respecto de la existencia de ganancias en habilidades de argumentación escrita a lo largo de un año de formación universitaria, en la Figura 1 se aprecia que existe una mínima ganancia en algunos subaspectos (fundamentación, aceptabilidad y suficiencia), la que es estadísticamente significativa para los sub-aspectos aceptabilidad $(t=-2,82, p<0,01,95 \%$ IC $[-0,46-0,08])$ y suficiencia $(t=-3,63, p$ $<0,0195 \%$ IC $[-0,46-0,13])$, que son los aspectos de calidad que inicialmente estaban más bajos. 
Figura 1: Puntaje promedio por subaspecto en ensayo 1 y ensayo 2

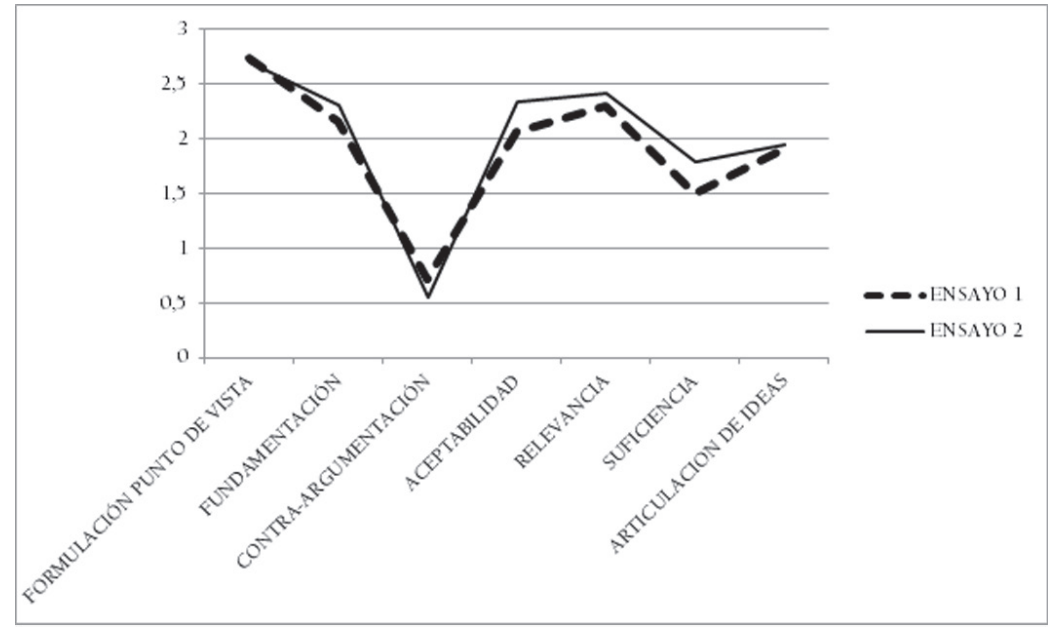

En la Tabla 5 se aprecia que los puntajes en los ensayos iniciales son mayores en el segundo año respecto del primero. Esto puede deberse tanto a un efecto de cohorte como a un impacto del año de educación superior.

Tabla 5: Descriptivos variables dependientes (muestra total según año)

\begin{tabular}{l|c|c|c|c|c|c|c|c|c}
\hline & \multicolumn{9}{|c|}{ Total } \\
\hline & \multicolumn{3}{|c|}{ Primer año } & \multicolumn{3}{c|}{ Segundo año } & \multicolumn{3}{c}{ Total } \\
\hline Variable & N & Media & DE & N & Media & DE & N & Media & DE \\
\hline Puntaje total ensayo 1 & 187 & 12,86 & 3,48 & 141 & 13,5 & 3,46 & 328 & 13,13 & 3,48 \\
\hline Puntaje total ensayo 2 & 112 & 13,41 & 3,36 & 84 & 14,6 & 2,4 & 211 & 13,9 & 3,13 \\
\hline Ganancia ensayo 1 y 2 & 58 & 0,72 & 4,13 & 44 & 0,5 & 3,49 & 102 & 0,62 & 3,85 \\
\hline Puntaje CALIDAD ensayo 2 & 112 & 6,16 & 2,09 & 84 & 6,93 & 1,27 & 211 & 6,49 & 1,85 \\
\hline Puntaje CALIDAD ensayo 1 & 187 & 5,48 & 2,08 & 141 & 5,85 & 2,03 & 328 & 5,64 & 2,06 \\
\hline Ganancia calidad & 58 & 0,68 & 2,82 & 44 & 0,68 & 1,96 & 102 & 0,68 & 2,47 \\
\hline $\begin{array}{l}\text { Puntaje ESTRUCTURA } \\
\text { ensayo 1 }\end{array}$ & 187 & 5,49 & 1,68 & 141 & 5,75 & 1,76 & 328 & 5,6 & 1,72 \\
\hline $\begin{array}{l}\text { Puntaje } \\
\text { ESTRUCTURA ensayo 2 }\end{array}$ & 112 & 5,30 & 1,62 & 84 & 5,71 & 1,76 & 211 & 5,47 & 1,64 \\
\hline \begin{tabular}{l} 
Ganancia en estructura \\
\hline
\end{tabular} & 58 & 0,06 & 1,95 & 44 & $-0,23$ & 2,24 & 102 & $-0,06$ & 2,07 \\
\hline
\end{tabular}


216 ¿LA UNIVERSIDAD EN CHILE PROMUEVE LAS HABILIDADES DE ARGUMENTACIÓN ESCRITA? UN ESTUDIO EXPLORATORIO COMPARATIVO DE ESTUDIANTES DE EDUCACIÓN UNIVERSITARIA Y EDUCACIÓN TÉCNICA - A. Larraín, P. Freire, R. Moretti, M.Requena y B. Sabat

Tabla 6: Ganancias entre ensayo 1 y 2 según carrera

\begin{tabular}{l|c|c|c|c|c|c|c|c|c}
\hline Carrera & $\mathrm{N}$ & Media & $\mathrm{DE}$ & $\mathrm{N}$ & Media & $\mathrm{DE}$ & $\mathrm{N}$ & Media & $\mathrm{DE}$ \\
\hline Derecho & 10 & 1,85 & 2,14 & 14 & $-0,46$ & 2,04 & 24 & 0,50 & 2,34 \\
\hline Psicología & 8 & 1,43 & 3,47 & 11 & 1,04 & 2,52 & 19 & 1,21 & 2,87 \\
\hline $\begin{array}{l}\text { Ing. Comercial } \\
\text { Asistente }\end{array}$ & 21 & 0,19 & 4,16 & 5 & $-0,8$ & 5,03 & 26 & 0 & 4,25 \\
\hline $\begin{array}{l}\text { Jurídico } \\
\begin{array}{l}\text { Asistente de } \\
\text { Párvulos }\end{array}\end{array}$ & 4 & 1,12 & 8,5 & 7 & 2,14 & 4,62 & 11 & 1,77 & 5,9 \\
\hline
\end{tabular}

En Derecho los estudiantes avanzaron bastante el primer año (especialmente en fundamentación, aceptabilidad y suficiencia. Ver Figura 2), pero el segundo año retrocedieron, mientras que en Técnico Jurídico los estudiantes avanzaron en ambos años, pero lo hicieron más pronunciadamente en el segundo, especialmente en los sub-aspectos fundamentación, aceptabilidad, relevancia y suficiencia (ver Figura 3).

Figura 2: Puntajes estudiantes carrera derecho ensayo 1 y 2 primer año

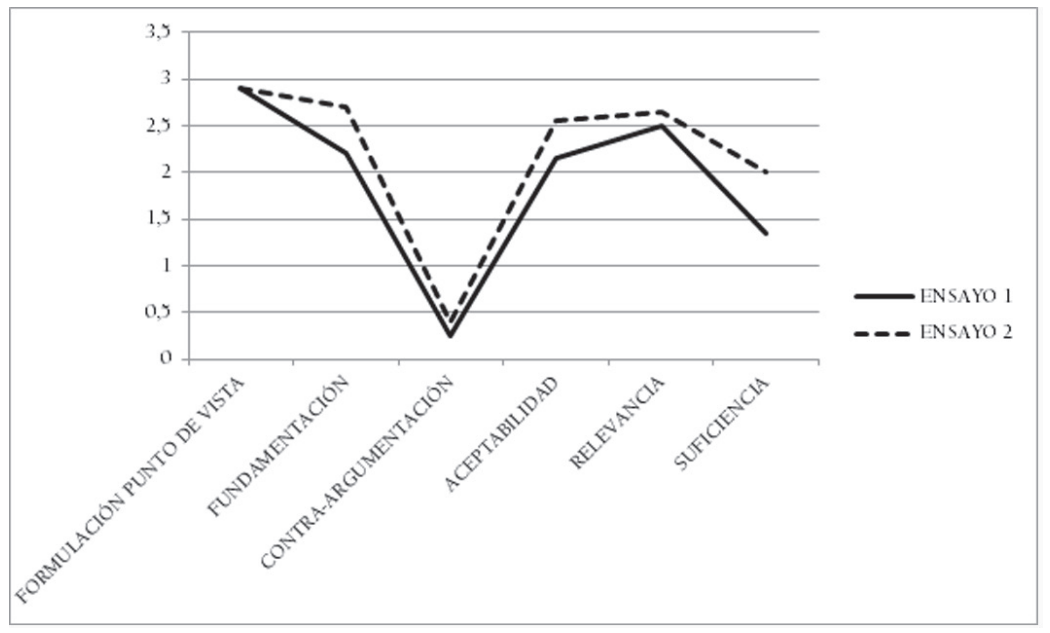


Figura 3: Puntajes estudiantes asistente jurídico ensayo 1 y 2 segundo año

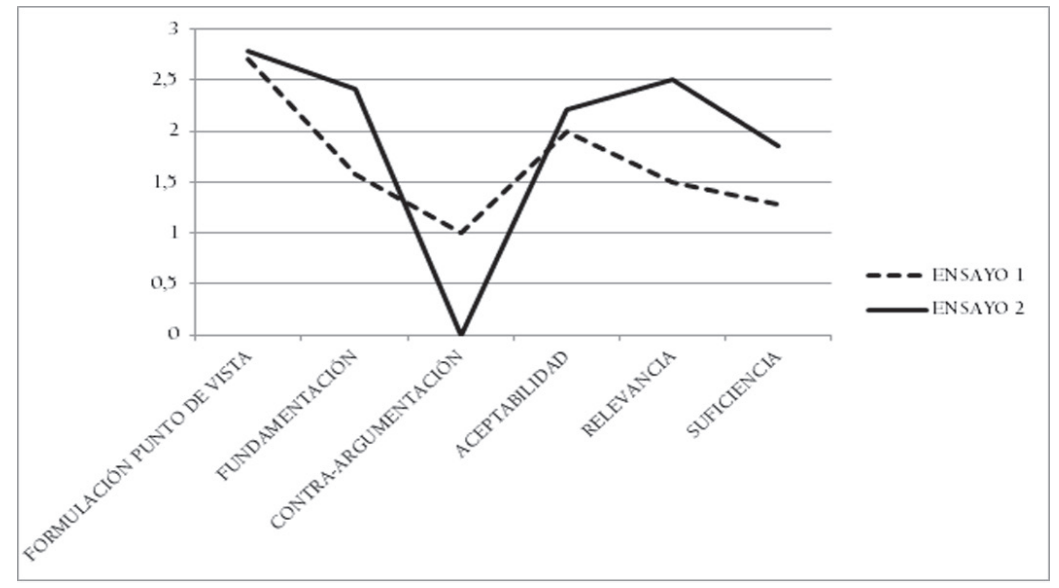

En Psicología ocurrió algo similar a Derecho: los avances se aprecian fundamentalmente en primer año en los sub-aspectos de fundamentación, aceptabilidad, relevancia y suficiencia (ver Figura 4).

Figura 4: Puntajes estudiantes psicología ensayo 1 y 2 primer año

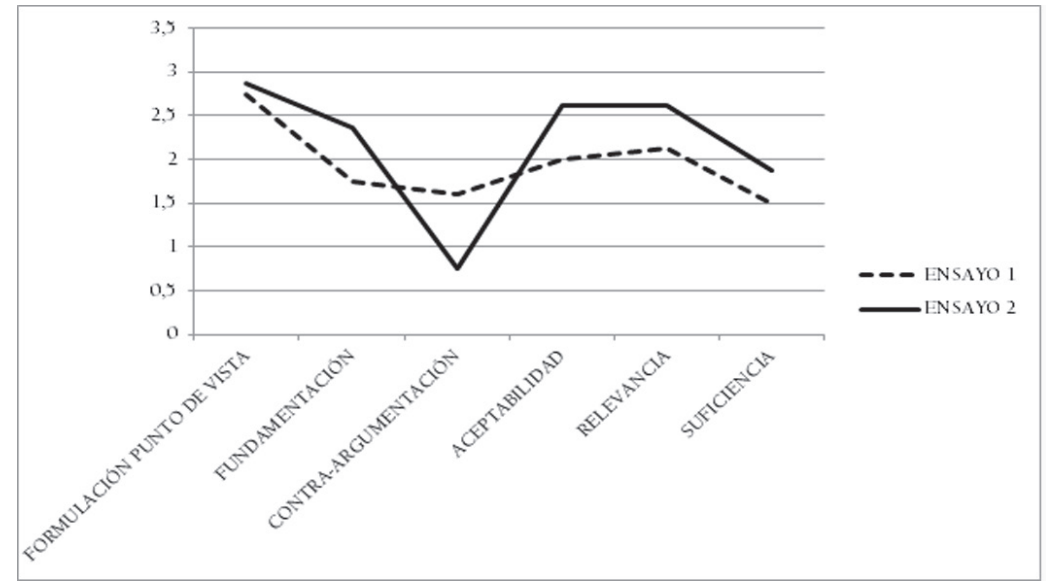

En Párvulos e Ingeniería Comercial los avances fueron bastante discretos: solo se aprecia un avance significativo en segundo año en los sub-aspectos de calidad (fundamentación en el caso de Asistente de Párvulos), pero un retroceso en los de estructura (ver Figura 5 y 6). Coherentemente, tanto en Párvulos como Ingeniería Comercial 
prácticamente no hay diferencias entre el primer y el segundo año, mientras que en las carreras que avanzaron sí las hay, siendo el promedio del ensayo 1 en estos casos mayor en el segundo año.

Figura 5: Puntajes estudiantes ingeniería comercial ensayo 1 y 2 segundo año

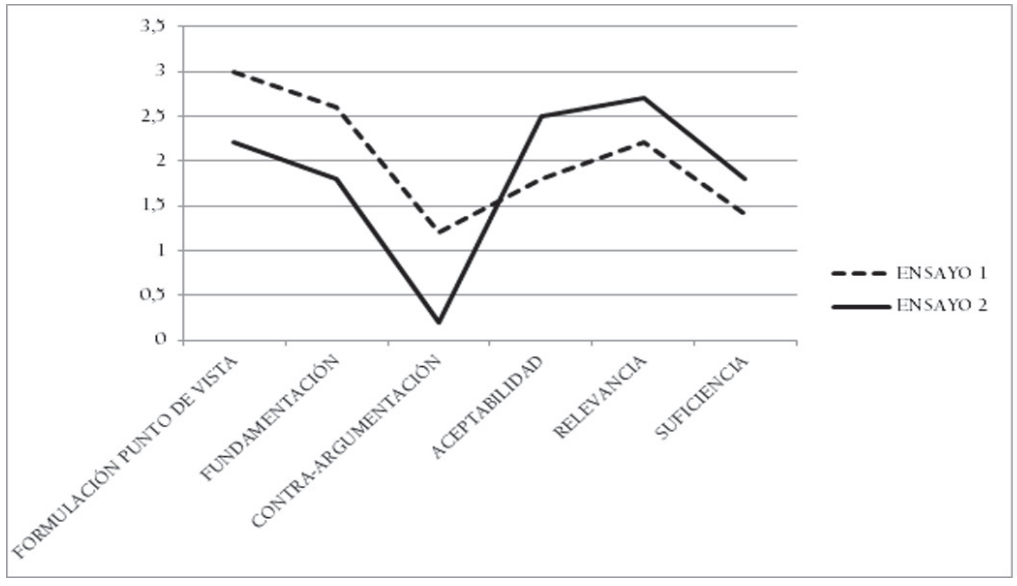

Figura 6: Puntajes estudiantes asistente de párvulos ensayo 1 y 2 segundo año

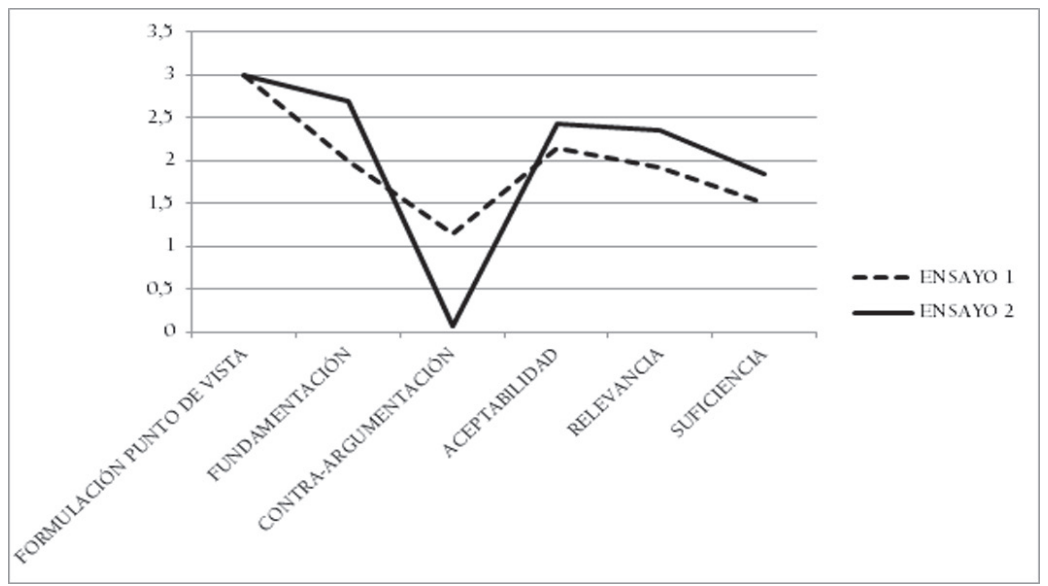

Se observa, entonces, una diferencia entre las ganancias de la universidad y las del instituto (ver Figura 7). Mientras en la primera se avanzó más en fundamentación, aceptabilidad y suficiencia, durante el primer año, en el instituto los estudiantes lo hicieron más en promedio en el segundo año y en la variable de relevancia. 
Figura 7: Diferencias entre ganancias universidad-instituto
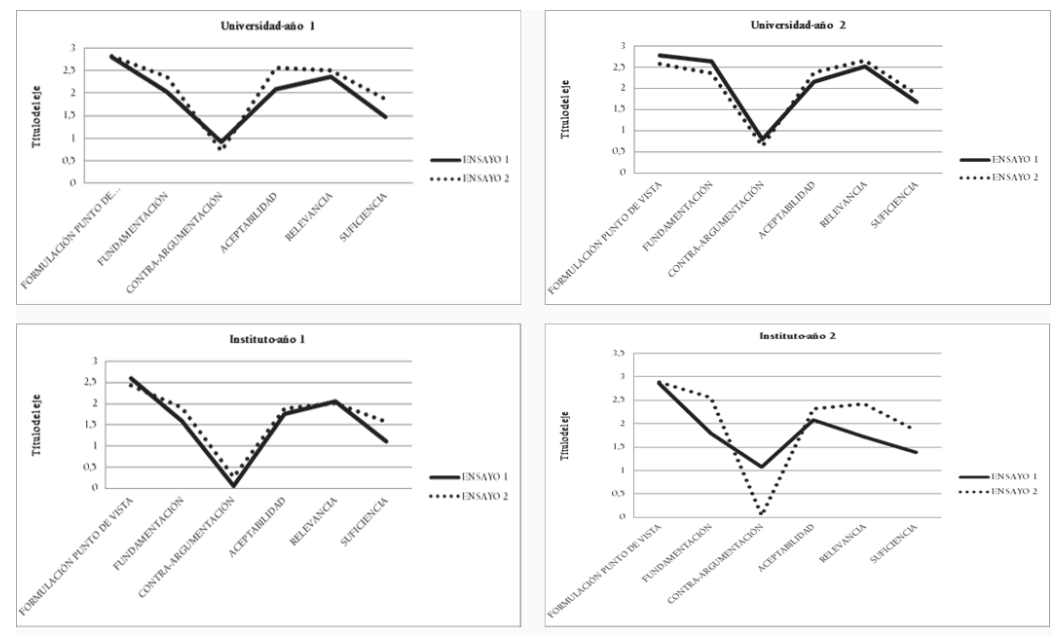

Complementariamente se realizaron análisis de correlaciones bivariadas entre los resultados de los dos ensayos y las medidas de rendimiento académico autorreportadas por los estudiantes en el cuestionario. Las únicas variables que correlacionan de manera estadísticamente significativa con el desempeño en los ensayos son el puntaje PSU (autorreportado) de Lenguaje (ensayo $1 r=330, p$ $=0,01$; ensayo $2 r=, 215, p=0,05) \mathrm{y}$, en el caso del ensayo 1 , las notas de enseñanza media (también autorreportadas) -NEM (ensayo $1 r=, 297, p=0,01$ ).

Para evaluar si las diferencias en ganancia de habilidades de argumentación escrita entre estudiantes universitarios y estudiantes no universitarios son estadísticamente significativas, se realizó un análisis de covarianza (ANCOVA). Los resultados mostraron que la diferencia es estadísticamente significativa al controlar por PSU de Lenguaje y edad ( $F=6,5, p<0,05$ IC 95\% [-13,28 7,46]) a favor de los estudiantes universitarios. Respecto del desempeño en el ensayo 1 , si se controla por las mismas variables hay un efecto estadísticamente significativo del año a favor de segundo año $(F=4,7, p<0,05$ IC $95 \%[-5,560,5])$. Variables socioeconómicas como dependencia del establecimiento educacional donde estudiaron su enseñanza escolar, o años de escolaridad de padre o madre, no mostraron tener un efecto principal en estas ganancias. 
Para evaluar si la ganancia en argumentación varía según si los estudiantes se encontraban sobre o bajo el promedio PSU de su cohorte, se realizó un ANOVA incluyendo factores como la carrera, año y ubicación en torno al promedio PSU. Los resultados mostraron una interacción estadísticamente significativa entre estos tres factores $(F=7,54, p<0,01)$, sugiriendo que la ganancia en argumentación varía según si los estudiantes están sobre o bajo el promedio PSU de su generación, pero lo hace diferencialmente según carrera y año. Por ejemplo, mientras los estudiantes de Derecho que se encuentran sobre el promedio PSU, aumentan $(M=606,23 \mathrm{DE}=56,27)$, en promedio 1,28 puntos en el ensayo en primero y retroceden casi un punto en segundo, los que están bajo el promedio lo hacen en 4,25 puntos en primero y aumentan un punto en segundo. En el caso de Ingeniería Comercial, mientras los estudiantes que se encuentran sobre el promedio PSU prácticamente no tienen ganancias en primero y retroceden 3,5 puntos en segundo, los que están bajo el promedio no tienen ganancias en primero, pero los de segundo avanzan ocho puntos. En el caso de Psicología, mientras los estudiantes que se encuentran, en promedio, sobre el promedio PSU no tienen ganancias en primero y avanzan 2,7 puntos en segundo, los que están bajo el promedio avanzan 4,5 puntos en primero, sin que se observe prácticamente avance en segundo. En el caso de Asistente Jurídico no hay estudiantes que se encuentren sobre el promedio PSU, y en promedio los estudiantes de primer año retroceden 4,5 puntos, mientras en segundo año ganan 1,4 puntos. Por último, los estudiantes de Asistente de Párvulos que se encuentran sobre el promedio PSU avanzan 1,25 puntos en primero y un punto en segundo, pero los que se encuentran bajo el promedio retroceden 1,5 puntos en segundo (no hay estudiantes sobre el promedio PSU en primer año).

\section{Discusión}

Los resultados son coherentes en distintos aspectos con la literatura existente y evidencia empírica disponible. Por una parte, los estudiantes muestran habilidades aceptables en cuanto a la formulación de argumentos, lo que es coherente con los estudios que muestran que estos avanzan en la vida escolar en la cantidad y complejidad de razones que usan para construir un argumento (Kuhn, 1996; Stein y 
Bernas, 1999). Coherentemente también con la evidencia empírica existente, la mayor debilidad de los estudiantes consiste en formular contraargumentos, lo que muestra que esta habilidad no está del todo desarrollada ni en los estudiantes universitarios ni en los técnicos al comienzo de la educación superior (Golder y Pouit, 1999). Respecto de los sub-aspectos de calidad, destaca la capacidad de incluir premisas relevantes y aceptables, pero sorprende el alto porcentaje de estudiantes que incluye premisas irrelevantes o negativamente relevantes, y poco aceptables. Debe llamar la atención en la misma línea, la baja capacidad de formular argumentos válidos usando premisas que sostengan suficientemente la conclusión. Esta aparece como la gran debilidad en términos de calidad argumentativa.

En síntesis, en términos de niveles de logro, se observa una tarea pendiente en cuanto a las habilidades argumentativas que muestran los estudiantes al inicio de la educación superior. Lo cual, más que una deuda del sistema universitario, es una deuda del sistema escolar. Aunque la argumentación es parte del marco curricular nacional y de los objetivos de aprendizaje de la enseñanza básica y media, estos no son completamente logrados. Es necesario reflexionar acerca de los modos en los que se está enseñando a argumentar en la escuela, pues la evidencia disponible muestra claramente que cuando se enseña a argumentar a los niños, niñas y jóvenes, estos aprenden y mejoran sustantivamente (Kuhn y Crowell, 2011; Kuhn y Udell, 2003; Piolat, Rousseay y Gombert, 1999; Zohar y Nemet, 2002).

Ahora bien, a juzgar por los resultados del estudio, se puede pensar que un año de enseñanza en educación superior en Chile tiene un efecto en el desarrollo de habilidades argumentativas, pero solo en los sub-aspectos de calidad, de aceptabilidad y suficiencia. Esto es importante, pues la suficiencia es un aspecto crucial y difícil de desarrollar. Sin embargo, ello no basta. Por un lado, la mejora en estos aspectos, aunque estadísticamente significativa, es muy pequeña y se verifica en los dos aspectos que estaban inicialmente más bajos. Por otro lado, la capacidad de formular contra-argumentos, que está íntimamente relacionada con habilidades metacognitivas y de pensamiento crítico (Leitão, 2000a), no mejora por la experiencia universitaria en los primeros años. De hecho, aunque en los aspectos de calidad y estructura 
los estudiantes de segundo año superan levemente a los de primero, esto solo se verifica para los puntajes en fundamentación, pero no en formulación de contra-argumentos. Así, aunque esta diferencia es estadísticamente significativa al controlar variables de entrada (lo que pudiera atribuirse a la experiencia de un año en educación superior), esto no redunda en mejoras en la formulación de contraargumentos. La evidencia muestra que este aspecto no se desarrolla solo por el paso del tiempo y, por el contrario, requiere dispositivos instruccionales complejos y sistemáticos, los que aparentemente no han estado presentes en la formación de estos jóvenes.

Es interesante notar que las ganancias en términos descriptivos varían según carreras en torno al año en que se producen. Esto puede sugerir que los avances realmente se deben a un efecto del plan de estudio en cada caso, y no simplemente a la experiencia universitaria en general. En el caso de Psicología y Derecho (a diferencia de lo reportado por Pascarella y Terenzini, 2005, y Reason, et al., 2006), los avances son más importantes en primer año, mientras que en Asistente Jurídico, Ingeniería Comercial y Párvulos, se verifica principalmente en el segundo año.

Por otro lado, la correlación entre el puntaje en los ensayos y el puntaje PSU de Lenguaje autorreportado, era esperable y es coherente con los resultados de los ensayos escritos que se realizan en la Pontificia Universidad Católica (Preiss, Castillo, Grigorenko, et al., 2013; Preiss, Castillo, Flotts, et al., 2013). El menor desempeño relativo en formulación de contraargumentos también se verifica en el caso de los ensayos de la PUC (Manzi y Flotts, 2012).

¿Las diferencias entre las ganancias de estudiantes universitarios y estudiantes no universitarios son significativas? Al controlar por PSU de Lenguaje y edad, la respuesta es que, en efecto, hay diferencias a favor de los estudiantes universitarios. Este dato es de suma relevancia, pues indica que hay un desarrollo de las habilidades de argumentación que no se explica simplemente por el desempeño previo, ni tampoco por la exposición a cualquier tipo de instrucción, sino por un efecto específico de la educación universitaria. Este efecto, que ha sido poco documentado, es coherente con los resultados de 
Kuhn (1996) que muestran que simplemente la edad no explica el desempeño argumentativo. Lo relevante aquí es la comparación entre distintos tipos de enseñanza superior, que no estaba presente en el estudio mencionado. Ahora bien, la pregunta es si es que esta ganancia argumentativa se debe a dispositivos pedagógicos o al efecto de la predominancia del discurso argumentativo en las evaluaciones universitarias (Andrews et al., 2006). Aunque en cualquier caso se trata de un efecto instruccional de la experiencia universitaria que sería distintivo respecto de la experiencia en educación técnica, es preciso avanzar en estudios que permitan saber si es que la educación universitaria se hace cargo de enseñar estas habilidades o simplemente las supone y demanda al momento de las evaluaciones de aprendizaje.

Por último, otro dato interesante es la diferencia en el desarrollo de habilidades de argumentación escrito en función de los estudiantes que están sobre o bajo el promedio de PSU. Con excepción de Asistente de Párvulos, los estudiantes que más avanzan son quienes reportan entrar a la educación superior con un desempeño más bajo que el promedio del grupo. Aunque es esperable, pues estos estudiantes tienen más espacio para avanzar, es relevante pensar que particularmente la enseñanza universitaria tiene cierta capacidad para hacer avanzar a los estudiantes que vienen más atrás en términos de conocimiento generales en Lenguaje y Matemática. Desde el punto de vista de la educación universitaria inclusiva, este dato es relevante, ya que no se trata simplemente de aceptar estudiantes con más dificultades académicas, sino de mostrar que se es capaz de compensar aquello que la educación escolar no entregó. En este sentido, aunque hay diferencias por carrera, es interesante notar que las habilidades argumentativas efectivamente pueden ser un indicador de aprendizaje universitario independientemente de las diferencias disciplinares. Así mismo, si bien es importante observar avances compensatorios en estudiantes con desempeños iniciales más bajos, se esperaría que todos avanzaran, pero eso no es lo que observamos en todos los casos, pues en algunas carreras el progreso de alumnos cuyo promedio PSU era superior al promedio del grupo fue casi nulo.

Creemos que es absolutamente necesario continuar investigando la capacidad de la enseñanza universitaria de aportar a las habilidades de 
argumentación de la población adulta en Chile, identificando procesos pedagógicos efectivos y experiencias exitosas. Este estudio es un primer paso que señala una tendencia que debe ser profundizada. Por una parte, es un estudio exploratorio en el que participan estudiantes de dos instituciones, por lo que las diferencias encontradas, más que deberse al tipo de formación ofrecida (universidad versus instituto), puede originarse en otro tipo de diferencia que puede verificarse entre instituciones de un mismo tipo. Por otro lado, cabe discutir el hecho de que la prueba se haya realizado por medio de un ensayo escrito. Como se señaló antes, está reportado que la argumentación escrita y la argumentación oral están íntimamente relacionadas. Sin embargo, es recomendable que futuras investigaciones acerca de la argumentación en estudiantes de educación superior consideren el estudio de las características de la argumentación oral en este grupo. Es posible hipotetizar que carreras como Periodismo puedan ser más fuertes en avance en argumentación escrita, mientras que Psicología y Derecho puedan ser más fuertes en argumentación oral.

Por último, cabe observar que las mujeres están sobrerrepresentadas (aproximadamente tres mujeres por cada dos hombres en el estudio), y los estudiantes técnicos son mayores en edad que los estudiantes universitarios. La diferencia numérica entre hombres y mujeres puede considerarse relevante dadas sus experiencias de socialización diferentes (asunto que rápidamente puede pensarse tras la ausencia de hombres en Asistente de Párvulos y en la fuerte feminización de carreras como Psicología). Por otro lado, la diferencia de edad entre técnicos y universitarios puede traducirse en diferencias normativas ligadas a la cohorte, las que pueden afectar los desempeños sin que ello sea detectable. En el caso de este estudio no indagamos en las trayectorias previas, lo que es claramente una limitación.

\section{Referencias}

Andrews, R., Bilbro, R., Mitchell, S., Peake, K., Prior, P., Robinson, A., See, B.H \& Torgerson, C. (2006). Argumentative skills in first year undergraduates: a pilot study, York: The Higher Education Academy.

Belland, B. R. (2010). Portraits of middle school students constructing evidence-based arguments during problem-based learning: the impact 
of computer-based scaffolds. Educational Technology Research and Development, 58(3), 285-309.

Carlino, P. (2005). Escribir, leer y aprender en la universidad. Una introducción a la alfabetización académica. Buenos Aires: Fondo de Cultura Económica.

Chen, C.H., \& She, H.C. (2012). The impact of recurrent on-line synchronous scientific argumentation on students' argumentation and conceptual change. Educational Technology E Society, 15 (1), 197-210.

Coirier, P., Andriessen, J., \& Chanquoy, L. (1999). From planning to translating: The specificity of argumentative writing. En J. Andriessen y P. Coirier (Eds.), Foundations of argumentative text processing (pp. 1-28). Amsterdam: Amsterdam University Press.

De Chiaro, S. \& Leitão, S. (2005). O papel do professor na construção discursiva da argumentação em sala de aula. Psicologia: Reflexão e Crítica, 18(3), 350-357. http://dx.doi.org/10.1590/s0102-79722005000300009

Garcia Mila, M. \& Andresen, C. (2008). Cognitive foundations of learning argumentation. En S. Erduran y M. P. Jimenez-Aleixandre (Eds.), Argumentation in science education: Perspectives from classroom-based research (pp. 29-46). Netherlands: Springer.

Glassner, A. \& Schwarz, B. B. (2005). The antilogos ability to evaluate information supporting arguments. Learning and Instruction, 15(4), 353-375. http://dx.doi.org/10.1016/j.learninstruc.2005.07.002

Golder, C. \& Pouit, D. (1999). For a debate to take place the topic must be debatable. Developmental evolution of the negotiation and debatability of arguments. En J. Andriessen y P. Coirier (Eds.), Foundations of argumentative text processing (pp. 137-148). Amsterdam: Amsterdam University Press.

Govier, T. (2010). A practical study of argument. Canada: Wardsworth.

Kuhn, D. (1996). Thinking as argument. En L. Smith (Ed.), Critical readings on Piaget (pp. 120-146). London: Routledge.

Kuhn, D. \& Crowell, A. (2011). Dialogic argumentation as a vehicle for developing young adolescents' thinking. Psychological Science, 22, 545552. http://dx.doi.org/10.1177/0956797611402512

Kuhn, D. \& Udell, W. (2003). The development of argument skills. Child Development, 74(5), 1245-1260. http://dx.doi.org/10.1111/14678624.00605

Leitão, S. (2000a). The potential of argument of knowledge building. Human Development, 43(6), 332-360. http://dx.doi.org/10.1159/000022695 
Leitão, S. (2000b). A produção de contra-argumentos na escrita infantil. Psicologia: Reflexão e Crítica, 13(3), 351-361. http://dx.doi.org/10.1590/ s0102-79722000000300004

Leitão, S. (2009). Arguing and learning. En C. Lightfoot y M. Lyra (Eds.), Challenges and strategies for studying human development in cultural contexts (pp. 221-251). Rome: Firera publishing.

Manzi, J. y Flotts, P. (2012). Medición de habilidades de comunicación escrita en estudiantes universitarios. La experiencia UC. Recuperado de http:// www.mideuc.cl/presentaciones_colmee/Paulina_Flotts_Examen_ Com_Escrita.pdf

Marinkovich, J. (2007). Las estrategias cognitivo-retóricas y la dimensión dialéctica de la argumentación oral en una clase de lengua castellana y comunicación. Revista Signos, 40, 127-146.

Mercier, H. \& Sperber, D. (2011). Why do humans reason? Arguments for an argumentative theory. Behavioral and Brain Sciences, 34, 57-11. http:// dx.doi.org/10.1017/S0140525X10000968

Mouraz, A., Leite, C., Trindade, R., Martins, J. M., Faustino, A. M., \& Villate, J. (2014). Argumentative skills in higher education: A comparative approach. Journal of Education \& Human Development, 3(1), 1-154.

O'Keefe, D. J. (1977). Two concepts of argument. Journal of the American Forensic Association, 13, 121-128.

Pascarella, E. T. \& Terenzini, P. T. (1991). How college affects students. San Francisco: Jossey- Bass.

Pascarella, E. T. \& Terenzini, P. T. (2005). How college affects students: A third decade of research, Volume 2. San Francisco: Jossey-Bass.

Peña, C. (2008). ¿Obsolescencia de la universidad moderna? Del conflicto de las facultades al capitalismo académico. En J. J. Brunner y Peña, C. (Eds.), Reforma de la educación superior. Santiago: Ediciones Universidad Diego Portales.

Piolat, A., Rossey, J. Y., \& Golmbert, A. (1999). The development of argumentative schema in writing. En J. Andriessen, M. Baker \& D. Suthers (Eds.), Arguing to learn: Confronting cognitions in computer supported collaborative learning environments. The Netherlands: Kluwe Academic Publishers.

Pirchio, S. \& Pontecorvo, C. (1997). Children's strategies in family disputes. Rassegna di Psicologia, 1, 83-106

Pontecorvo, C. \& Pirchio, S. (2000). A developmental view on children's arguing: The need of the other. Human Development, 43(6), 361-363. http://dx.doi.org/10.1159/000022696 
Preiss, D. D., Castillo, J. C., Flotts, P., \& San Martín, E. (2013). Assessment of argumentative writing and critical thinking in higher education: Educational correlates and gender differences. Learning and Individual Differences, 28, 193-203. http://dx.doi.org/10.1016/j. lindif.2013.06.004

Preiss, D. D., Castillo, J. C., Grigorenko, E., \& Manzi, J. (2013). Argumentative writing and academic achievement: A longitudinal study. Learning and Individual Differences, 28, 204-211. http://dx.doi.org/10.1016/j. lindif.2012.12.013

Reason, R. D., Terenzini, P. T., \& Domingo, R. J. (2006). First things first: Developing academic competence in the first year of college. Research in Higher Education, 47(2), 149-175. http://dx.doi.org/10.1007/s11162005-8884-4

Rojas-Drummond, S. \& Peon Zapata, M. (2004). Exploratory talk, argumentation and reasoning in Mexican primary school children. Language and Education, 18(6), 539-557. http://dx.doi. org/10.1080/09500780408666900

Santos, C. M. \& Santos, S. L. (1999). Good argument, content and contextual dimensions. En J. Andriessen y P. Coirier (Eds.), Foundations of argumentative text processing (pp. 75-95). Amsterdam: Amsterdam University Press.Scholtz, Z. Sadek, M., Hodges, M., Lubben, F., \& Braund, M. (2006). Argumentation about data: Learners' ability to think critically. C. Malcom (Ed.), Proceeding of the 14th Annual Conference of the South African Association for Research in Mathematics, Science and Technology Educaction (pp. 658-664). South Africa: SAAEMSTRE,

Song, Y. \& Ferreti, R. (2013). Teaching critical questions about argumentation through the revising process: Effects of strategy instruction on college students' argumentative essays. Reading and Writing: An Interdisciplinary Journal, 26(1), 67-90. http://dx.doi.org/10.1007/s11145-012-9381-8

Stein, N. \& Albro, E. (2001). The origins and nature of arguments: Studies in conflict understanding, emotion, and negotiation. Discourse Processes, 32(2-3), 113-133. http://dx.doi.org/10.1080/016385 3X.2001.9651594

Stein, L. N. \& Bernas, R. S. (1999). The early emergence of argumentative knowledge skills. En G. Rijlaarsdam y E. Espéret (Series Eds.) y J. Andriessen y P. Coirier (Vol. Eds.), Studies in writing: Vol. 5. Foundations of argumentative text processing (pp. 97-116). Amsterdam: Amsterdam University Press.

Stein, N. \& Miller, C. A. (1993). A theory of argumentative understanding: Relationships among position preference, judgments of goodness, 
memory, and reasoning. Argumentation, 7(2), 183-204. http://dx.doi. org/10.1007/bf00710664

Sunal, C., Sunal, D., \& Tirri, K. (abril, 2001). Using evidence in scientific reasoning: Exploring characteristics of middle school students' argumentation. Trabajo presentado en el encuentro anual de la American Educational Research Association, Seattle, Washington.

Terenzini, P. T., Springer, L., Yaeger, P. M., Pascarella, E. T., \& Nora, A. (1996). First generation college students: Characteristics, experiences, and cognitive development. Research in Higher Education, 37(1), 1-22. http://dx.doi.org/10.1007/bf01680039

Terenzini, P. T. (2010). El egresado, reflejo de su institución educativa. En Calidad de los egresados, responsabilidad institucional ineludible. Seminario internacional 2009. Santiago: Consejo Nacional de Educación/Comisión Nacional de Acreditación.

Walker, C. M., Wartenberg T. E., \& Winner E. (2013). Engagement in philosophical dialogue facilitates children's reasoning about subjectivity. Developmental Psychology, 49(7), 1.338-1.347. http:// dx.doi.org/10.1037/a0029870

Wilson, C. D., Taylor, J. A., Kowalski, S. M., \& Carlson, J. (2010). The relative effects and equity of inquiry-based and commonplace science teaching on students' knowledge, reasoning, and argumentation. Journal of Research in Science Teaching, 47(3), 276-301. http://dx.doi. org/10.1002/tea.20329

Zohar, A. \& Nemet, F. (2002). Fostering students knowledge and argumentation skills through dilemmas in human genetics. Journal of Research in Science Teaching, 39(1), 35-62.

Recibido: 06/07/2015

Aceptado: 03/12/2015 